\title{
Early curing age paste backfill exposures - the role of effective stress
}

\author{
RL Veenstra Glencore Australia Pty Ltd, Australia \\ AG Grice AMC Mining Consultants (Canada) Ltd., Canada \\ MW Grabinsky University of Toronto, Canada \\ WF Bawden Mine Design Engineering, Canada
}

\begin{abstract}
This paper presents results from a numerical modelling exercise that looks at vertically exposing sub-28-day cemented paste backfill. The reason for this exercise is that underground mining operations would like to start exposing cemented paste backfill (CPB) earlier in order to increase production. However, these earlier exposures would mean that the pore water pressures in the stope may not have fully dissipated, hence entering an effective stress state versus a total stress state. The goal of this paper was to examine how effective stress impacts these early exposures. This was done by modelling the filling and the subsequent pore water dissipation within a stope. This modelled CPB was then vertically exposed, using both effective and total stress models, at various times ranging from immediately after filling to 28 days after filling. The results of these models were then compared in order to determine what difference there was between the overall outcome.
\end{abstract}

\section{Introduction}

The backfilling of voided stopes is an important part of some underground mines' extraction sequence because it provides regional ground support and allows for maximal ore recovery. Cemented paste backfill has become a popular backfilling method due to its delivery speed, versatility, and engineered strength.

Maximal ore recovery requires the CPB to be vertically exposed when an adjacent stope is blasted and mucked. This exposure requires the CPB to be strong enough to prevent it from failing into the recently voided stope. In order to gain this strength, the cementing agent within the CPB needs to cure for a certain period of time.

This time period ranges but is usually at least 28 days, a typical curing period adopted from the cement and concrete industry. However, there has been a recent trend to decrease this curing age in order to extract stopes earlier in the schedule with the hope of increasing mine productivity. These earlier exposures reduce the CPB's curing time and could take place prior to the full dissipation of the pore water pressure (PWP) in the stope.

This paper examines what role effective stress plays in these proposed early exposures of CPB. The first part of the paper will examine field instrumentation from a series of test stopes to determine PWP dissipation rates for several CPB streams. The authors conducted a numerical modelling study to replicate the filling of the stope and the dissipation of the PWP post-filling. The backfill within the numerical stope was then exposed at a series of curing ages between the end of filling and the end of the PWP dissipation.

It is currently industry practice to model vertical exposures based on total stress analytical solutions. This numerical exercise compares the results from effective and total stress exposure models in order to determine what differences there are between the model results. An analysis was performed to determine what impact these differences have on the final model outcome. 


\section{Field instrumentation}

In the last decade there have been several large instrumentation programs undertaken to study the in situ behaviour of CPB within a series of test stopes (Grabinsky et al. 2008; Thompson et al. 2011; Hasan et al. 2014). The instrumentation in these studies varied but almost all the instrumentation clusters included the use of three total earth pressure cells (TEPCS) and a piezometer (PZ). Figure 1 contains three photographs of a typical instrumentation cluster installation in the University of Toronto (UofT) test stopes. Refer to Thompson et al. (2011) for more details about the UofT in situ instrumentation.
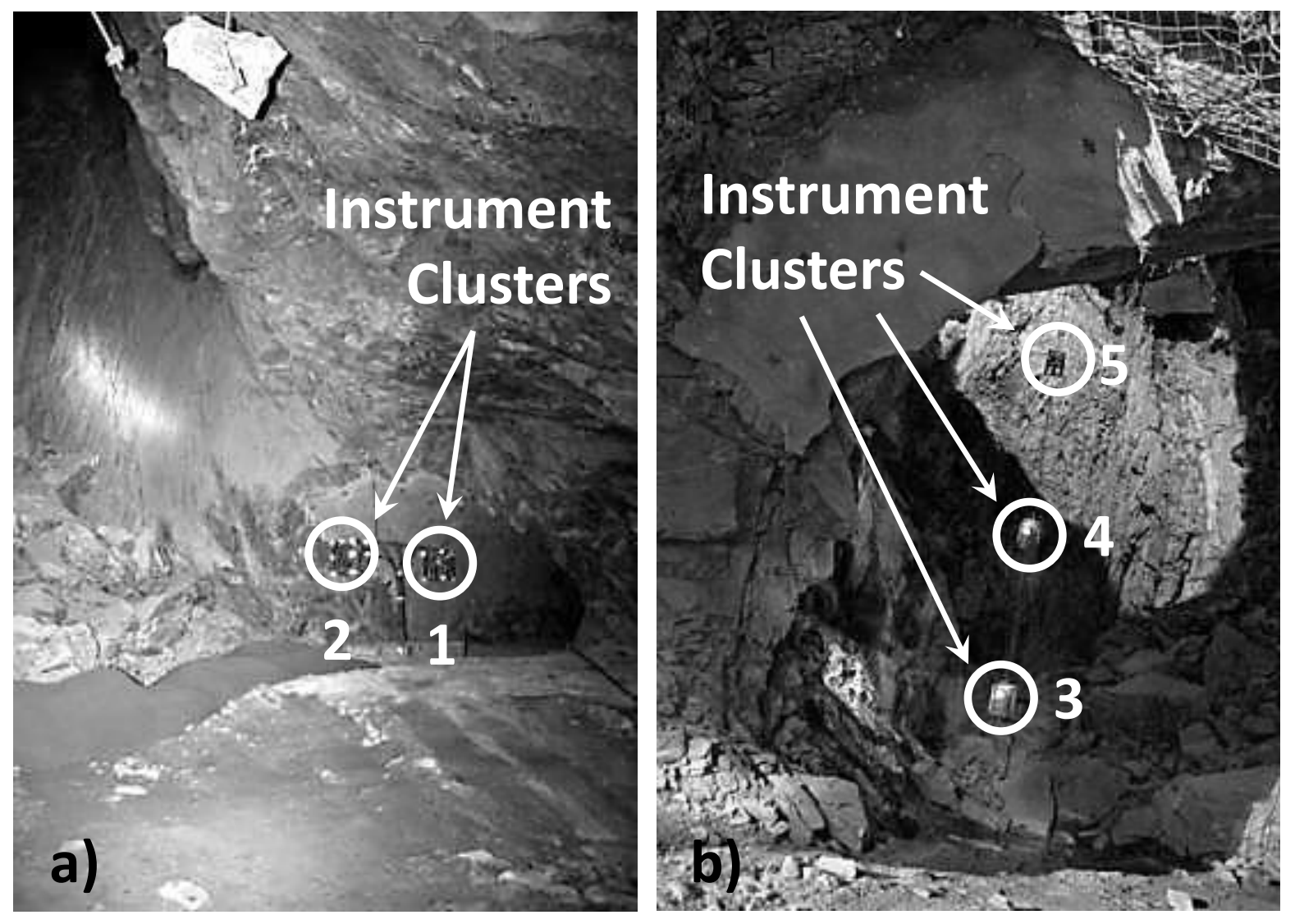

Figure 1 Photographs showing the installation of instrumentation at the (a) brow; and (b) stope body

Instrumentation from three different UofT test stopes are analysed in this paper. Table 1 provides a summary of the stopes and their respective instrumentation clusters. For example, the table details whether the instrument cluster was encased in high or low strength CPB and if there was any lengthy pauses ( $>5$ hours) during filling.

\section{Table 1 Summary of test stopes' instrument clusters}

\begin{tabular}{cccc}
\hline Stope & Instrument cluster & CPB strength & Filling regime \\
\hline 1 & 3 & Filled with one & $\sim 70$ hour pause in pour between \\
& 4 & CPB stream & 20 and 90 hours \\
2 & 4 & High & $\sim 12$ hour pause between 36 and \\
& 5 & Low & 48 hours
\end{tabular}




\begin{tabular}{llll}
2 & High & \\
3 & High & Continuous \\
& 4 & Low & \\
\hline
\end{tabular}

Figure 2 shows the in situ instrumentation results for three of the UofT test stopes. In these graphs, instrument cluster numbers of three or higher indicate that these clusters were installed in the stope body (Figure 1(b)), with the instrument cluster 3 being the closest to the bottom of the stope. Figure 2(c) contains data from a number two instrument cluster, which was installed at the brow of the stope (Figure 1(a)).
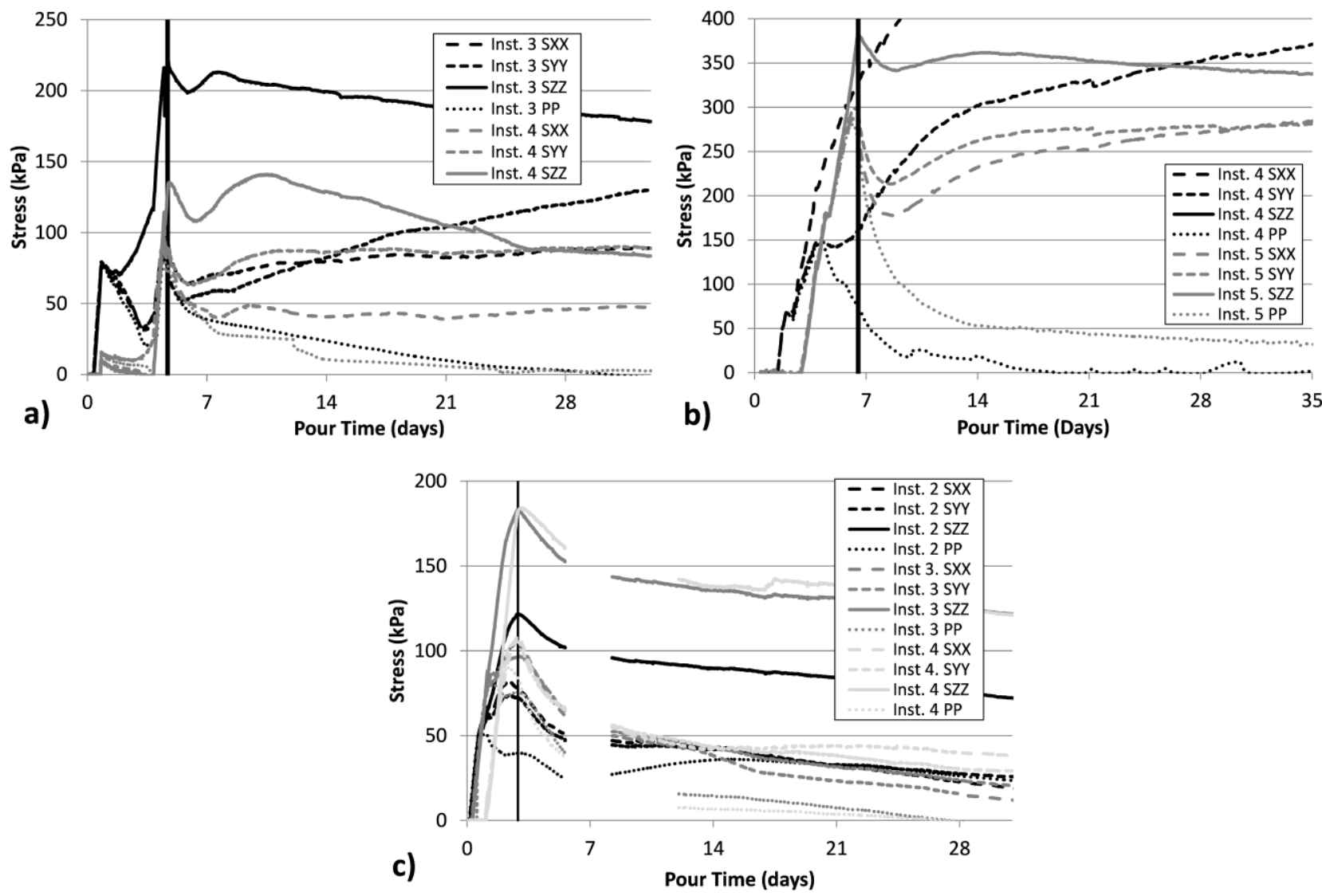

\section{Figure 2 Instrumentation plots for (a) stope 1; (b) stope 2; and (c) stope 3}

All of the graphs in Figure 2 contain a thick, vertically oriented black line. This indicates when the stope had finished filling. The graph then shows the next 28 days' worth of instrumentation data. Note that in Figure 2(b) that the SZZ stress of the 4th instrument cluster is missing due to an instrumentation malfunction.

All three plots show similar trends with the pressures remaining isotropic for an initial period of time and then starting to diverge. It is interesting to note that the PWPs from the instrument clusters encased in high strength CPB generally start to deviate prior to the end of the pour. However, all of the instrument clusters show that once the filling stops the PWPs start to dissipate. In the case of Figure 2(a), this happens twice: once during the extended pour pause, and again when the stope has been fully backfilled. Note that the PWP dissipation response is similar in both cases.

Table 2 summarises the PWP dissipation times as determined from the instrumentation. Note that there are many reasons why these dissipation times can vary (cement hydration, drainage paths, tailings types etc.). However, the majority of dissipation times are similar to each other. 
Table 2 Summary of instrumentation PWP dissipation times

\begin{tabular}{cccc}
\hline Stope & Instrument cluster & CPB strength & PWP dissipation time \\
\hline \multirow{2}{*}{1} & 3 & Filled with one & $20-23$ days \\
& 4 & CPB stream & \\
2 & 4 & High & $\sim 14$ days \\
& 5 & Low & $>28$ days \\
& 2 & High & $>28$ days \\
3 & 3 & High & $>28$ days \\
& 4 & Low & $>28$ days \\
\hline
\end{tabular}

A dissipation time of approximately 28 days will be used as a guideline for the modelling presented in this paper.

\section{$3 \quad$ Modelling}

The modelling was completed on a 2D $18 \times 30 \mathrm{~m}$ mesh. The stope was modelled using a numerical code based in Itasca's Flac3D numerical software (Itasca Consulting Group, Inc. 2014). For more information on this code, refer to Veenstra (2013) or Veenstra et al. (2014). However, the model used in this paper has additional cut-off logic applied to the fluid-flow boundary conditions.

\subsection{Methodology}

The model was filled using a $0.3 \mathrm{~m} / \mathrm{h}$ rise rate, which meant a filling time of 100 hours. The model was then run for a further 28 days ( 670 hours) to dissipate the PWP. This meant that the model was run for a total of 770 hours. In order to examine the exposure behaviour of the backfill, it was exposed at five different exposure times: zero days (100 hours of runtime), three days ( 170 hours of runtime), seven days ( 270 hours of runtime), 14 days ( $\sim 40$ hours of runtime), and 28 days ( $\sim 70$ hours of runtime).

Two different exposure models were used: an effective stress model with the PWPs maintained and a total stress model with the PWPs removed. This was done in order to compare how the total and effective stress models behave when exposed. Note that the effective stress exposure models were run in an undrained state.

The CPB at all curing ages was modelled as a perfectly plastic Mohr-Coulomb material. An isotropic fluid-flow model was used. The derivation of the required parameters for these models is discussed in the next section.

\subsection{Input parameters}

The model input parameters were generated by testing conducted at UofT (Mozaffaridana 2011; Veenstra 2013). It consisted of unconfined compressive strength (UCS) and direct shear box testing. The shear box testing allowed the early age ( $<48$ hour) strengths to be determined while the UCS testing allowed the later strengths to be determined. The friction angle of the CPB was assumed to be a constant $35^{\circ}$. The shear box results were converted to UCS using the relationship below.

$$
\sigma_{c}=\frac{2 c(\cos \phi)}{1-\sin \phi}
$$


Where:

$$
\begin{aligned}
& \sigma_{c} \quad=\text { equivalent UCS. } \\
& c \quad=\text { material's cohesion. } \\
& \phi \quad=\text { material's friction angle. }
\end{aligned}
$$

The reconciliation of the results from the two testing methods is shown in Figure 3(a). The results these tests were also used to calculate or estimate the Young's modulus values at different curing ages (Figure 1(b)). Finally, the model also requires permeability. Two permeability curves are shown in Figure $1(\mathrm{c})$. The first is the permeability as calculated from the laboratory testing and the second was the modified permeability curve used in the modelling.
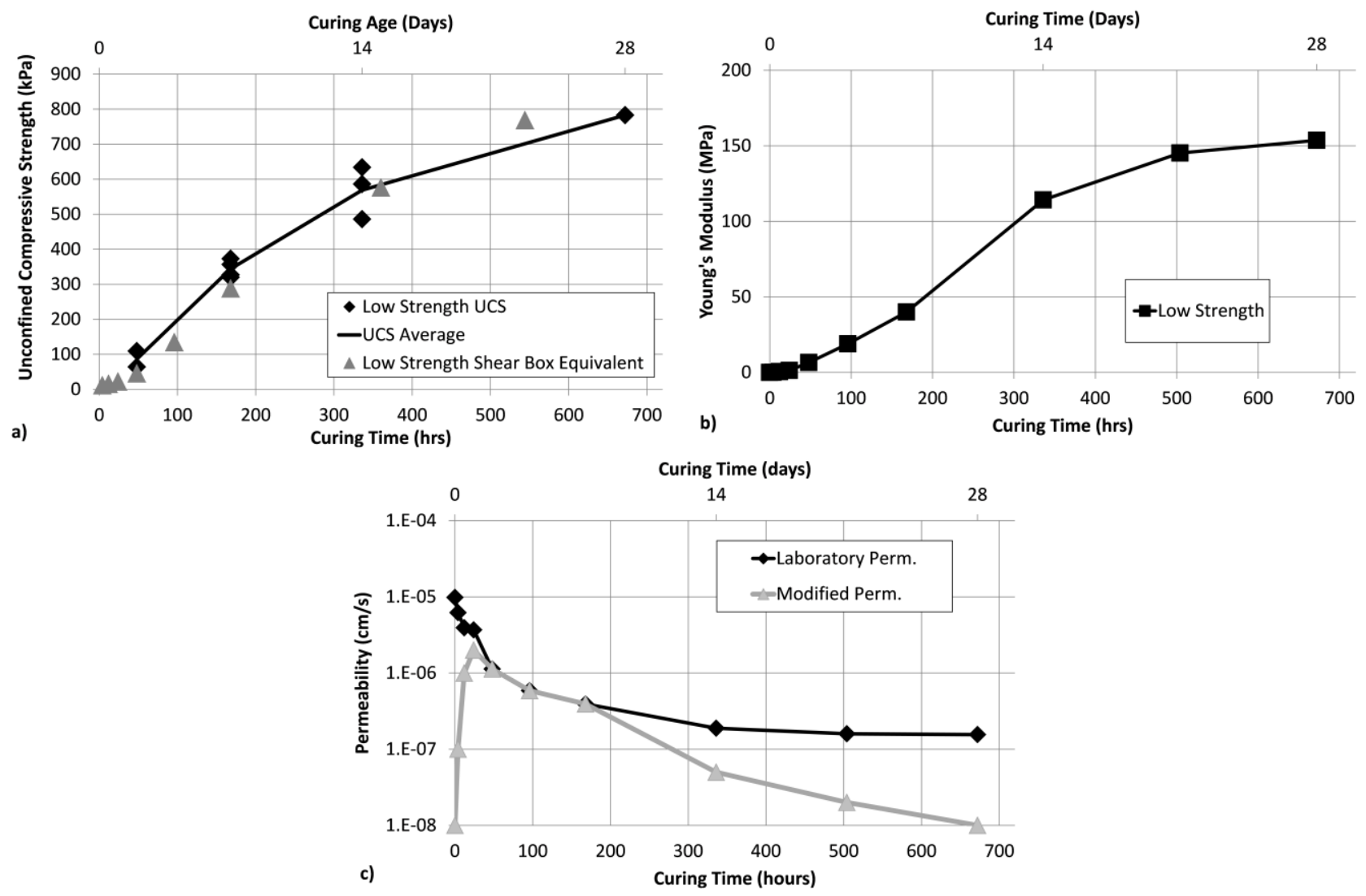

Figure 3 Laboratory testing results for (a) unconfined compressive strength; (b) Young's modulus; and (c) permeability

\subsection{Results}

This section presents the results of the pre-exposure and exposure models.

\subsubsection{Pre-exposure}

Figure 4 contains model contour plots showing total vertical stress, PWP and effective vertical stress. The total stress plots also show the location of three monitoring points where the model recorded the stress and PWP. These recorded values were plotted in the graph seen in the figure.

The contour plots, in Figure 4, show the increase in effective stress well. As the PWPs dissipate the difference between the total and effective stress contour plots decreases. This is readily apparent in the similar appearance of the non-PWP contour plots of the 400 and 770 hour models. 
This graph shows that the data from the model monitoring points generally follows the trends observed in the instrumentation results examined in Section 2. All three monitoring points show initially isotropic stresses. The stresses at the lower point start to deviate prior to the completion of filling while the other stresses at the higher points remain isotropic during the filling. After filling the monitoring points, all show stress decreases that are similar to those observed in the field instrumentation.

Note that the PWPs do not fully dissipate in the model but, in most cases, have reached a steady state sometime prior to the end of the 28-day dissipation period. The exception is the PWP from the middle monitoring point, which was still decreasing. The lack of complete PWP dissipation is due to both the permeability of the material as well as the fluid-flow boundary conditions. However, there was enough similarity between the field instrumentation and the pre-exposure model results to allow the use of the pre-exposure model results as a starting point to model the vertical exposures.

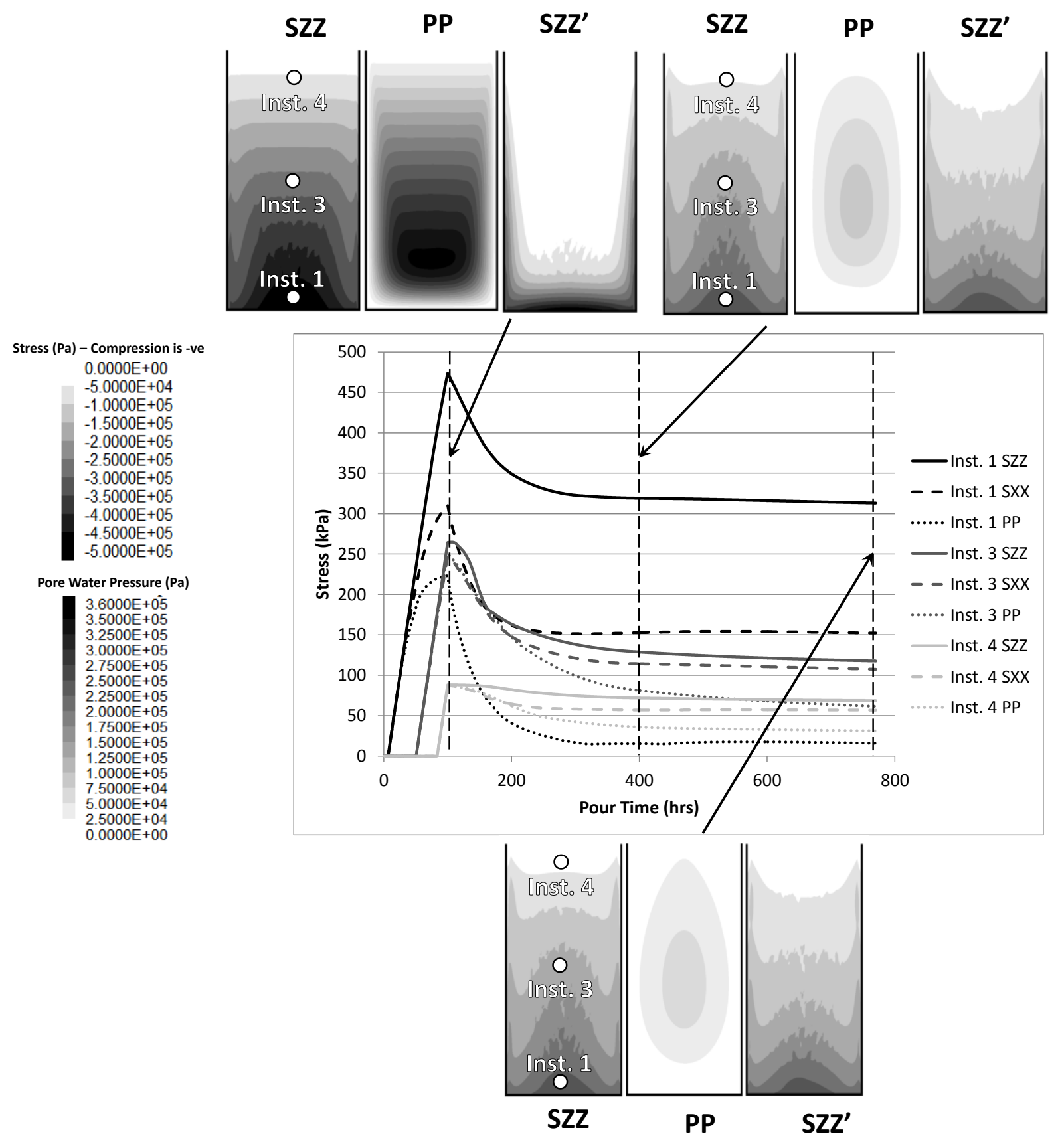

Figure 4 Model contour plots showing total vertical stress, pore water pressure, and effective vertical stress at pour times of 100,400 , and 770 hours 


\subsubsection{Exposure}

When CPB is exposed, there are three different types of failure patterns: negligible, surface and deep. These are shown schematically in Figure 5. The main difference between the three patterns is the size of the failures and the underlying mechanics. The negligible and surface failures are related to the change in stresses at the exposure edge due to relaxation derived from the release of confinement. The deep failure is more akin to a gravity driven slope failure.

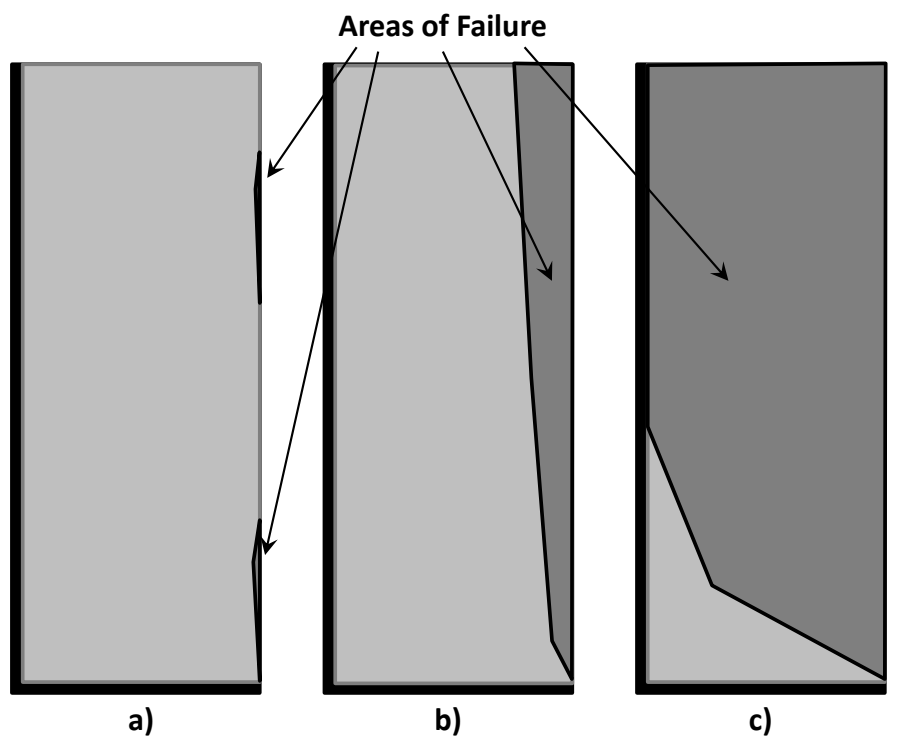

\section{Figure 5 Schematic showing (a) negligible; (b) surface; and (c) deep failure patterns of a vertical exposure in $\mathrm{CPB}$}

\subsubsection{Initial failure mechanisms}

A comparison between the total and effective stress models determined that they had different initial failure mechanisms. The failure mechanisms for the effective stress models are shown in Figure 6 . The plots in this figure are from the model exposed immediately upon completion of filling. Figure 6(a) shows the pre-exposure effective vertical stress (SZZ'). Comparatively, Figure 6(b) shows the SZZ' immediately after exposure. Note that contours have shifted and that the effective stress is starting to reduce on the right-hand side (RHS) of the stope. Figure 6 (c) shows the post exposure contours with the failed elements. Note that all of the models' zone states were reset prior to exposure in order to examine only the failure caused by the exposure. These plots show that there are tensile failures throughout the stope in the approximate shape of the effective stress zone. However, the exposed side of the stope also has large amounts of sheared zones indicating that the RHS material is progressively failing. Note also the surficial failure at the lower RHS of the stope. 


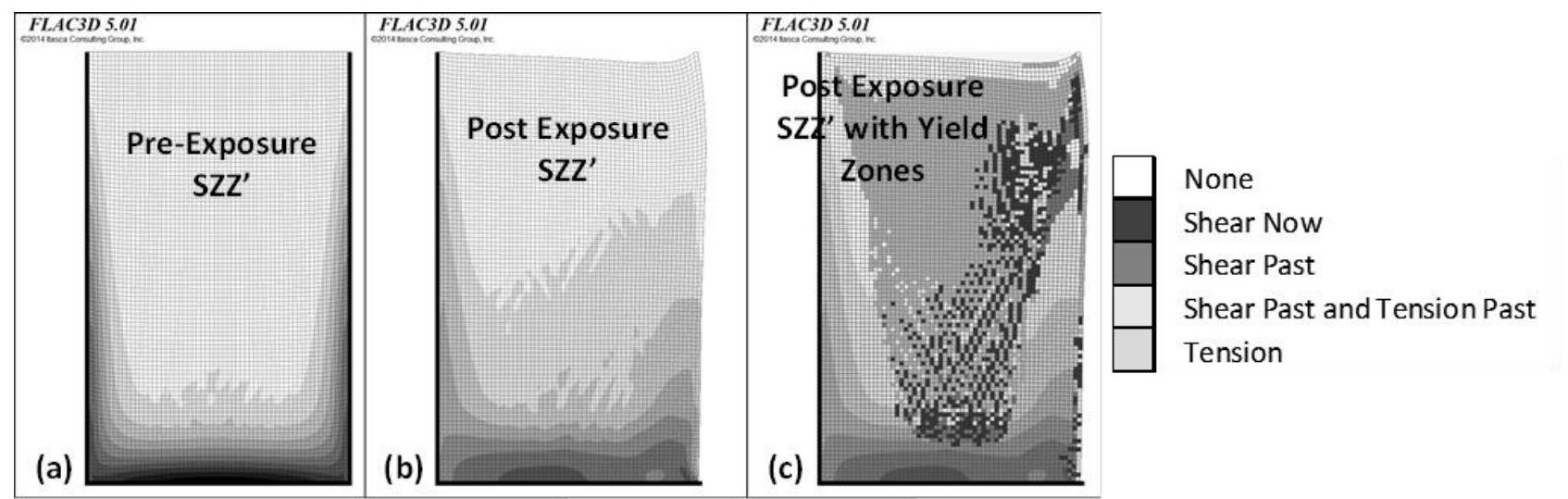

\section{Figure 6 Vertical effective stress contour plots for (a) pre-exposure; (b) immediately post exposure; and (c) with yield zones overlain the post exposure (mesh deformation at $+5 \%$ )}

This failure mechanism is consistent in all of the effective stress models. This mechanism initiates with tensile yielding in the zero effective stress zone. The weight of this failed mass is placed onto the RHS of the low effective stress zone, which then starts to fail in shear. However, as illustrated in Figure 7, the location of these failures change with increased exposure times. These changes are due to the decreasing size of the low effective stress zone with time and the zone's migration towards the top of the stope, as well as the increased strength of the CPB with time.

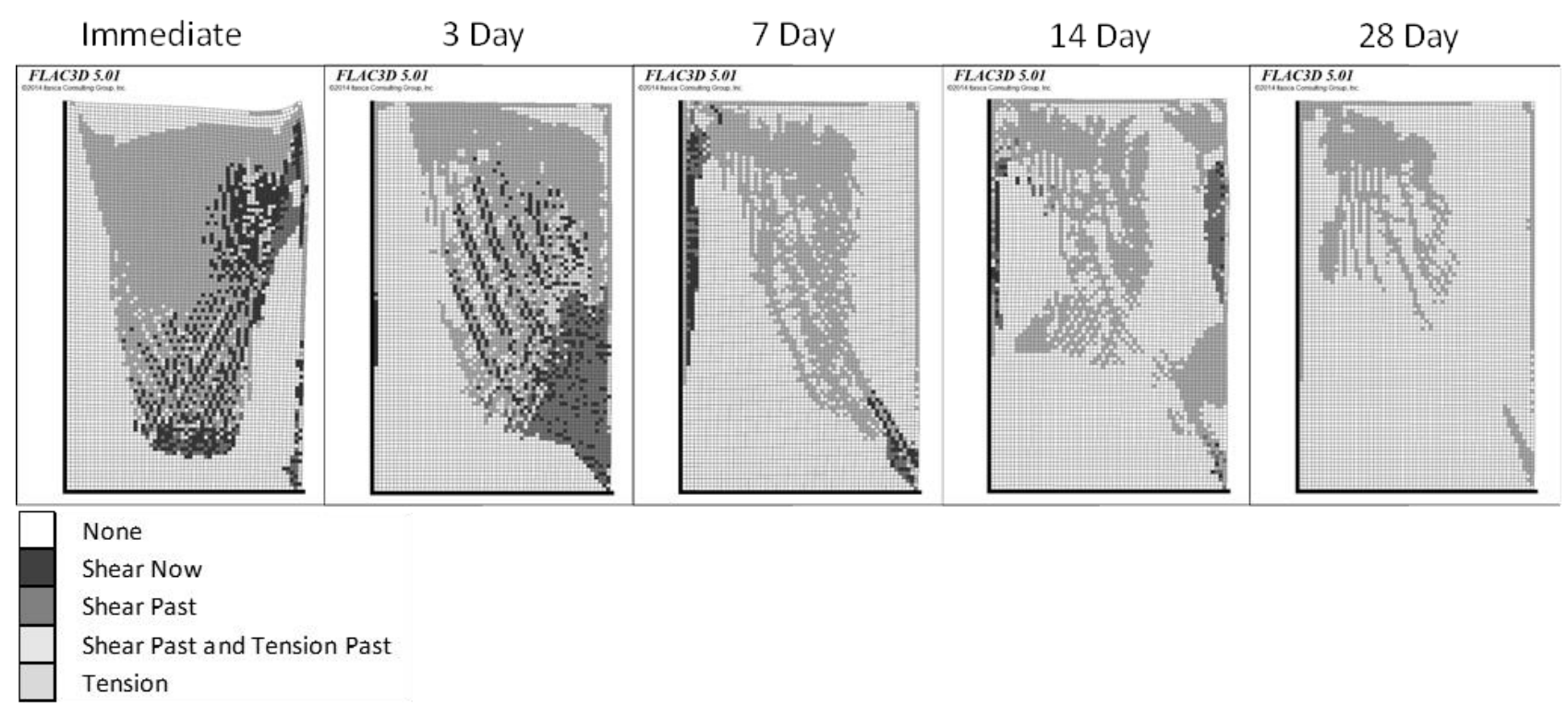

\section{Figure 7 Zone state change between effective stress models at different exposure times (mesh deformation at $+5 \%$ )}

The immediate and three-day exposure models show large central failure zones with areas of progressively failing fill at the surface of the exposure. However, as the exposure time increases it is observed that initial tensile failure within the low effective stress zone continues but that the progressive failure has been replaced by the development of a shear band at the lower section of the exposure. The size and length of this band also decreases with increased exposure time. These types of failures can be classified, particularly at the shorter exposure times, as deep failures as per Figure 5(a).

The effective stress model plots can be compared to the total stress plots for the same exposure times (Figure 8). The plots in this figure indicate that majority of the failure is tensile due to the release of confinement due to the CPB's exposure (Figure 5(b)). This tensile failure front then migrates away from the 
exposure surface. There is secondary tensile failure front that migrates from the upper side of unexposed side of the stope due to the overall displacement of the CPB towards the exposure. All of the models develop some shear failures, but these failures are generally restricted to the immediate surface of the exposure. The immediate exposure model is an exception as there are shear failures through the failing areas of the fill. This is due to the low strength of the fill at this time.

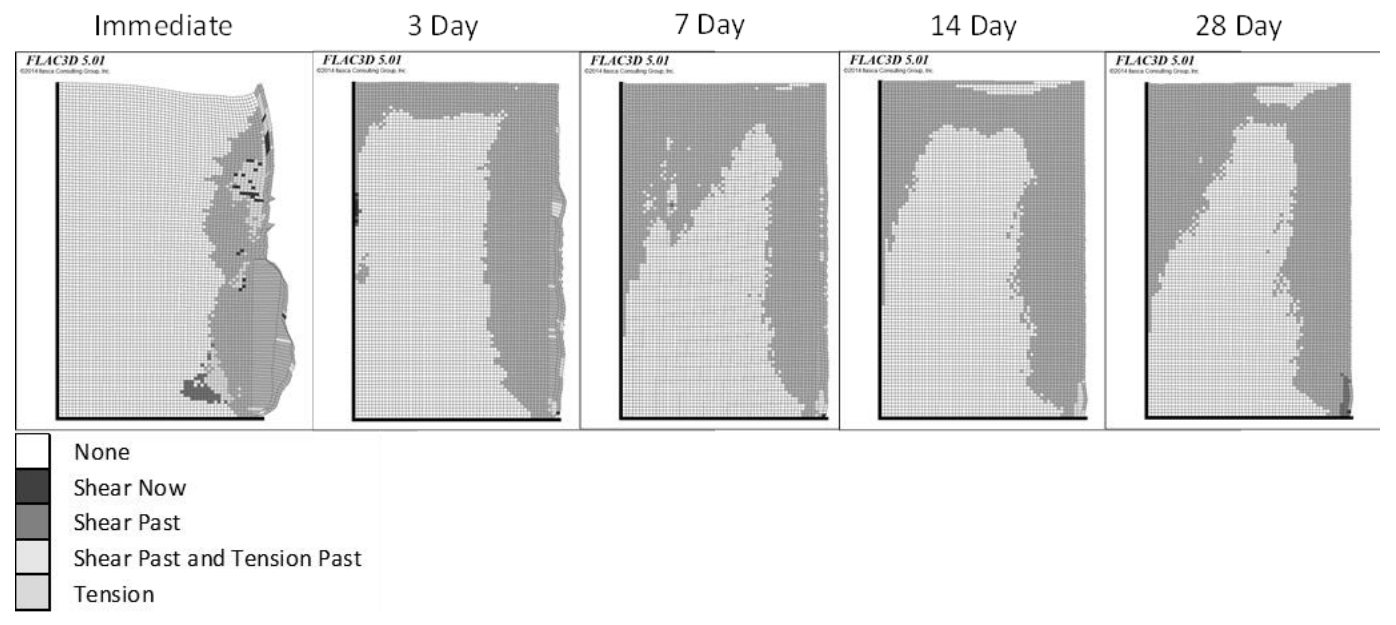

\section{Figure 8 Yield zone change between total stress models at different exposure times (mesh deformation at $+5 \%$ )}

This analysis has shown that the failure mechanisms for the effective and total stress models are different. The next step is to examine whether or not these differences affect the end result of the model.

\subsubsection{Final model outcome}

Figure 9 shows the each mode's zone state after the model had been exposed and then run for a further 200,000 time steps. The effective stress models are on the top and the total stress models are on the bottom. An estimated failure surface for each model has been drawn on the plots. These surfaces were estimated based on the strains, displacements, and velocities calculated by the model and the locations of these surfaces were used to classify the type of failure that each exposure generated. For example, both the total and effective immediate models contained deep failures, whereas the 14-day effective stress model was a deep failure compared to negligible failures observed in the total stress model. 


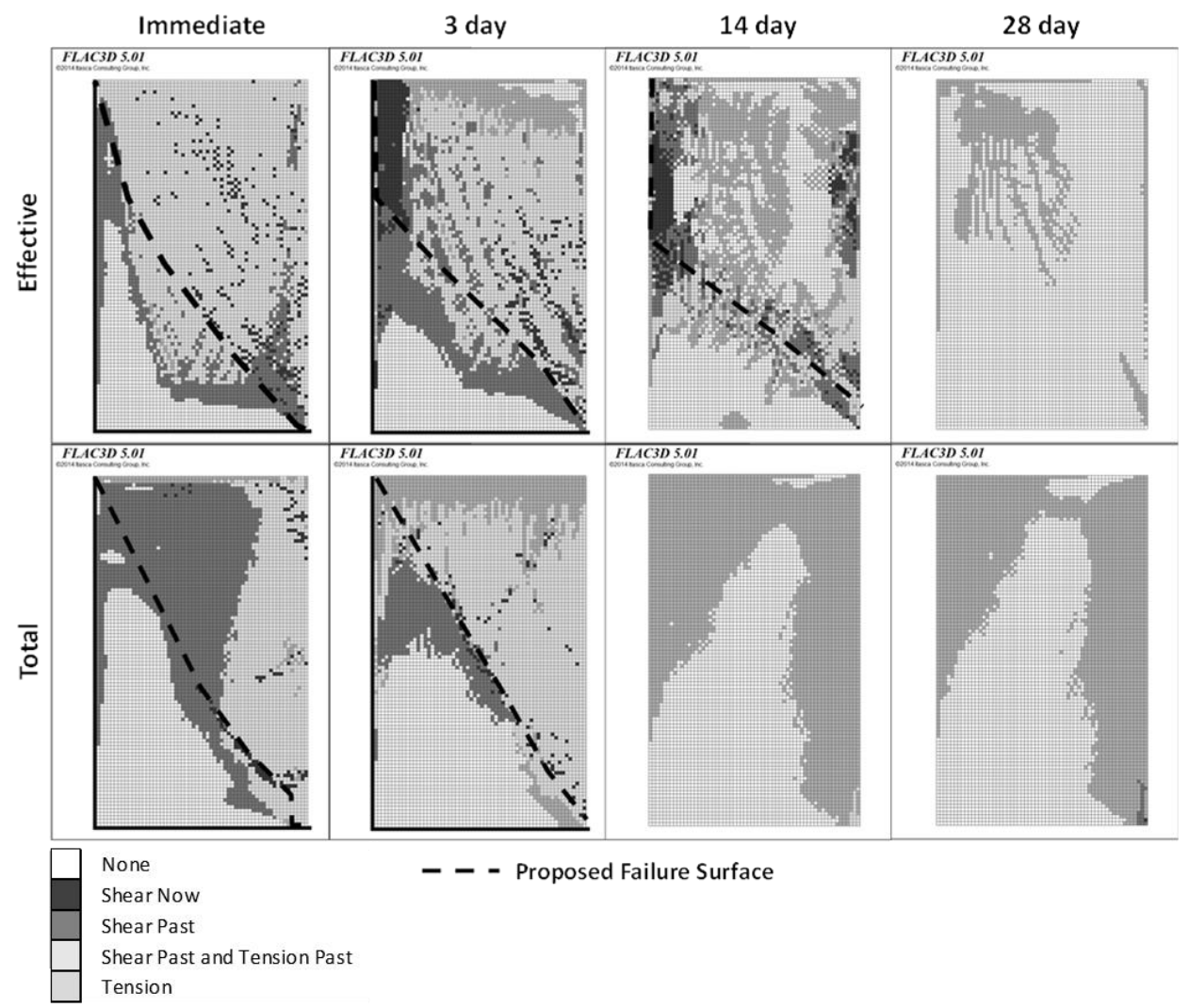

Figure 9 Final model zone states 200,000 model steps after exposure 

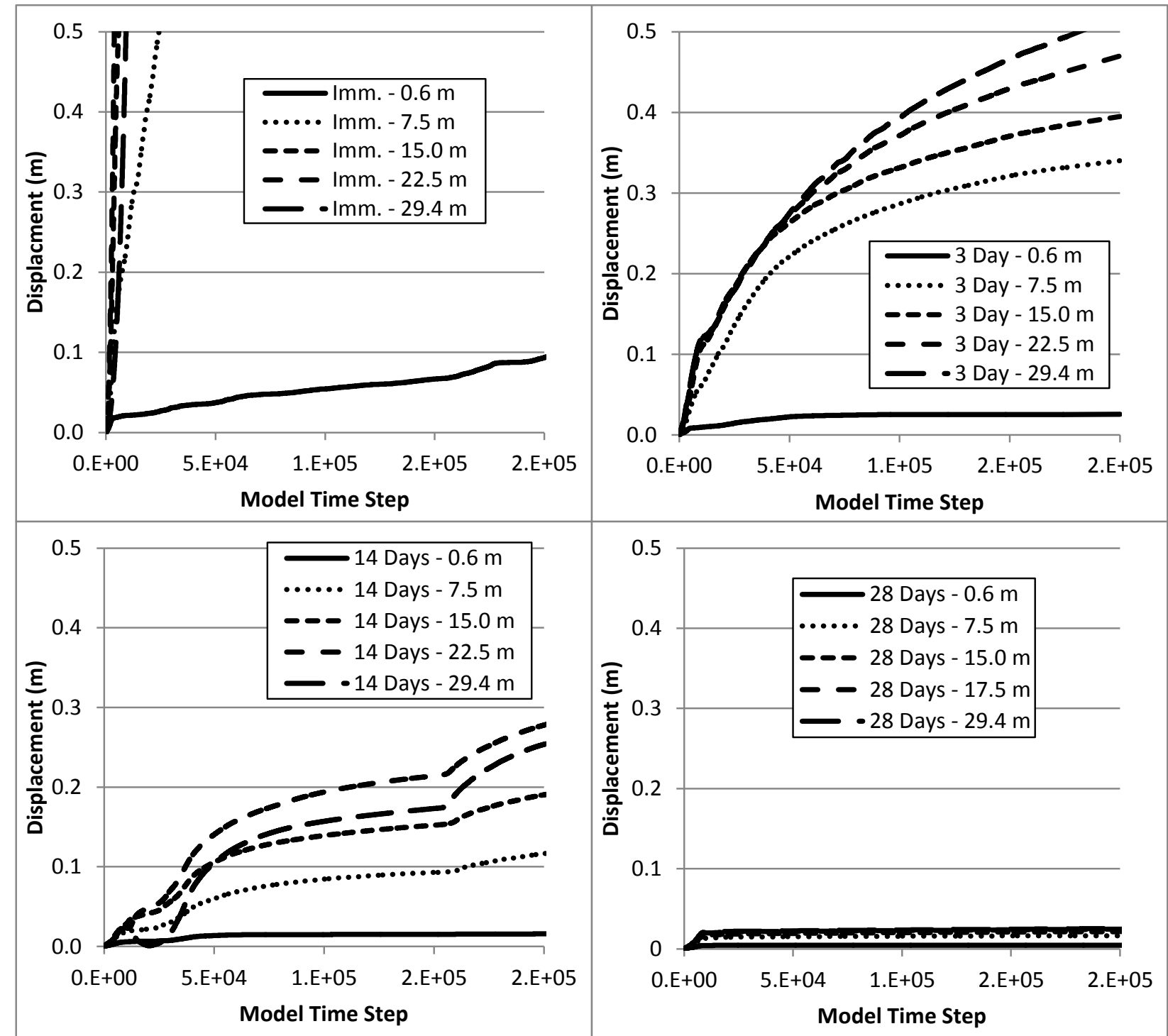

\section{Figure 10 Graphs showing the displacement along the exposure height for the immediate, three-day, 14-day, and 28-day exposure effective stress models}

Figure 10 contains four displacement plots showing data obtained from the model monitoring points along the height of the exposure. Note that lengths shown in the plot are from the bottom of the stope. These plots show displacements from the immediate, three-day, 14-day, and 28-day effective stress exposure models. These plots can be used to determine the relative speed of progressive failure. For example, the immediate exposure plot shows that the top four monitoring points rapidly exceed displacements of $0.5 \mathrm{~m}$ whereas the displacements in the 28-day plots showed very little movement. The other plots (three and 14-day) show a slow creeping type of failure. Note that the 14-day plot shows an interesting stepping behaviour. These steps correspond to the 'linking' of different shear failure areas into one larger failure plane.

\subsection{Discussion}

Table 3 summarises the stope models in terms of failure type, failure progression, the amount of PWP dissipation, UCS and the difference between the total and effective stress models. Note that the speeds given in the failure progression row are related only to the model's internal time step and not an actual real world time. 
Table 3 Summary of exposure stability

\begin{tabular}{|c|c|c|c|c|c|c|c|c|c|c|}
\hline Exposure time & Immed & iate & Three- & day & Seven & -day & 14-d & & 28-d & \\
\hline Model type & Effective & Total & Effective & Total & Effective & Total & Effective & Total & Effective & Total \\
\hline $\begin{array}{c}\text { Failure type } \\
\text { (Figure 5) }\end{array}$ & Deep & Deep & Deep & Deep & Deep & Surface & Deep & Neg. & Neg. & Neg. \\
\hline $\begin{array}{l}\text { Failure } \\
\text { progression } \\
\text { (in terms of } \\
\text { model time } \\
\quad \text { step) }\end{array}$ & Fast & Fast & Fast & Med. & Med. & Fast & Slow & Fast & Slow & Fast \\
\hline $\begin{array}{l}\text { Total PWP } \\
\text { dissipation }\end{array}$ & & & & & & & & & & \\
\hline $\begin{array}{c}\text { (in \% of middle } \\
\text { monitoring } \\
\text { location) }\end{array}$ & 0 & & 40 & & 74 & & 92 & & 10 & \\
\hline UCS (kPa) & 200 & & 350 & & 50 & & $64 C$ & & 775 & \\
\hline $\begin{array}{c}\text { Difference in } \\
\text { model } \\
\text { outcome }\end{array}$ & Non & & Med & & Hig & & $\mathrm{Hig}$ & & Non & \\
\hline
\end{tabular}

Note: Neg. = negligible; Med. = medium

This table highlights that the largest difference between the effective and total stress models were at seven and 14 days. This corresponds to approximately $40-90 \%$ of the total PWP dissipation. This is important, as this is the range where most mines would typically want to push their exposure times. The table also highlights that early age (immediate and three-day) and the 28-day model have limited differences in results. The early results are likely due to the lower strengths of the CPB while the CPB at 28 days is the strongest as well as having the highest PWP dissipation.

The table also suggests that allowing early exposures of CPB may not be a trivial undertaking for a mining operation. The procedure for allowing earlier exposures would involve a specific laboratory testing program as well as detailed numerical modelling in order to capture the individuality of a particular mine's CPB properties and stope geometry. It would also be important to establish a monitoring program for these exposures when they are exposed.

\section{Conclusions}

The goal of this paper was to determine what affect the PWP pressure had on vertical exposure of sub-28-day cured paste. This stems from a common question asked by mining operations looking to increase production by reducing stope cycle times. In order to accomplish this goal, a Flac3D model was used to first mimic filling a stope with CPB and second to model the dissipation of the PWPs within the stope. The results of this modelling were compared to in situ instrumentation data and both showed similar trends.

This model was then used as a basis for two exposure models: an effective stress model and total stress model. The CPB was exposed at five times: immediately, three days, seven days, 14 days, and 28 days. These modelling results were then analysed based on failure method, failure progression, and the difference in overall outcome between the models. This analysis showed the failure mechanisms of the two models were different. Despite this difference, the early age models (immediate and three-day) and the 28-day models had similar overall model results. However, analysis of the seven and 14-day results showed that there was a large difference in the overall model results. 
The early age results are attributed to the low UCS values of the CPB at these early curing times (i.e. the PWP makes no difference, as the CPB is too weak to remain stable). The 28-day results are due to the CPB being the strongest as well as having the most PWP dissipation. The remaining times are in middle ground where the CPB is weak enough to still be affected by the PWP. This middle ground is important as this is the typical time period where mining operations are looking to start exposing CPB filled stopes. It also indicates that the question of early exposures is not trivial and would require additional study if a mining operation was intent on pursuing such a course.

\section{References}

Grabinsky, MW, Bawden, WF, Simon, D, \& Thompson, B 2008, 'In situ properties of cemented paste backfill in an Alimak stope', Proceedings of the 61st Canadian Geotechnical Conference, Canadian Geotechnical Society, Edmonton.

Hasan, A, Suazo, G, Doherty, JP \& Fourie, AB 2014, 'In situ measurements of cemented paste backfill in an operation stope at Lanfranchi Mine', in Y Potvin \& AG Grice (eds), Proceedings of the 11th International Symposium on Mining with Backfill, Australian Centre for Geomechanics, Perth, pp. 327-336.

Itasca Consulting Group, Inc. 2014, 'FLAC3D: Fast Lagrangian Analysis of Continua in 3 Dimensions', version 5.10, Itasca Consulting Group, Inc., Minneapolis, http://www.itascacg.com/software/flac3d

Mozaffaridana, M 2011, 'Using thermal profiles of cemented paste backfill to predict strength', Master's thesis, University of Toronto.

Thompson, BD, Grabinsky, MW, Veenstra, RL \& Bawden, WF 2011, 'In situ pressures in cemented paste backfill - a review of fieldwork from three mines', in RJ Jewell \& AB Fourie (eds), Proceedings of the 14th International Seminar on Paste and Thickened Tailings, Australian Centre for Geomechanics, Perth, pp. 491-504.

Veenstra, RL 2013, 'A design procedure for determining the in situ stresses of early age cemented paste backfill', PhD thesis, University of Toronto.

Veenstra, RL, Grabinsky, Bawden, WF \& Thompson, BD 2014, 'The use of numerical modelling to determine the stress within early age cemented paste used to backfill an underground stope', in Y Potvin \& AG Grice (eds), Proceedings of the 11th International Symposium on Mining with Backfill, Australian Centre for Geomechanics, Perth, pp. 97-112. 